\title{
Mini-Review
}

\section{Pathogenic and immunosuppressive properties of mycobacterial phenolic glycolipids}

Reid Oldenburg ${ }^{1,2,3}$, Caroline Demangel $^{1,2 *}$

${ }^{1}$ Institut Pasteur, Unité d'Immunobiologie de l'Infection, Paris, France

${ }^{2}$ INSERM U1221, Paris, France

${ }^{3}$ Université Paris Diderot, Sorbonne Paris-Cité, Paris, France

*Corresponding author: demangel@pasteur.fr; Institut Pasteur, Unité d'Immunobiologie de I'Infection, 25 Rue Du Dr Roux, 75724 Paris Cedex 15, France. 


\section{ABSTRACT}

Phenolic glycolipids (PGL) are polyketide synthase products that are uniquely produced by a subset of pathogenic mycobacteria and are displayed at the bacterial cell surface, in a strategic position to interfere with host immune cells. Their expression has been associated with enhanced mycobacterial virulence in vivo, and suppression of the inflammatory responses of host phagocytes in vitro. In this review, we will present our current understanding of the mode of operation of PGL, along with functional evidence that demonstrates the evolutionary advantage conferred by PGL production for host cell invasion, intracellular persistence and evasion of host immune and bactericidal responses. 


\section{Introduction}

Mycobacterium is a genus of Actinobacteria, the sole member of the Mycobactericeae family in the order Actinomycetales (1). Mycobacteria can be subdivided into fast- and slow-growing species, a subdivision that is recapitulated by $16 \mathrm{~S}$ rRNA sequence homology analysis across the genus $(2,3)$ (Figure 1). Most mycobacterial species are innocuous saprophytes living in the soil, water, plants and in the air, however the slow-growing species subfamily contains major pathogens for humans. Mycobacterium tuberculosis and the genetically-related members of the M. tuberculosis complex ( $M$. canetti, M. africanum, M. microti and M. bovis) cause tuberculosis in humans and other animals (4). M. tuberculosis is considered to be one of the most successful pathogens on the planet, with about one third of the human population infected worldwide (5). M. leprae causes leprosy, the second most common mycobacterial disease in humans. Of note, the anti-tuberculous vaccine $M$. bovis Bacille Calmette Guérin (BCG) was generated empirically by Calmette and Guérin by serial passages of a virulent strain of $M$. bovis in bile salts (6-8). Aside from its use as a live vaccine, and a treatment for superficial bladder cancer, BCG represents a useful vector for the delivery of heterologous antigens in vivo. It also provides researchers with a genetically engineerable reference strain for the study of species-specific products, such as phenolic glycolipids (PGL) (9).

The mycobacterial cell wall is unique among bacteria due to its lipid-rich, exceptionally impermeable structure (10-12). From interior to exterior, this cell wall consists of a plasma membrane and an outer, Gram-negative-like membrane (called mycomembrane) containing mycolic acids (MA). Besides MA, the mycomembrane also contains non-covalently bound lipids such as phthiocerol dimycocerosates (DIM), polyacyl trehaloses (PAT), diacyltrehaloses (DAT), sulfoglycolipids (SGL), lipomannan (LM) and lipoarabinomannan (LAM). In addition, the mycomembrane is coated by a detergent-labile capsule containing arabinomannan, alpha-glucan and oligomannosyl-capped glycolipids and proteins, which together with the cell wall forms the mycobacterial cell envelope. While the architecture and 
composition of this envelope is largely conserved within the Mycobacterium genus, individual lipid constituents can differ across strains or clinical isolates. This is well exemplified by PGL, a family of polyketide synthase-derived, cell surface-displayed lipids that are only found in a limited subset of pathogenic species (Figure 1) $(13,14)$. Structurally, mycobacterial PGL all contain a phenol ring that is linked to a DIM-related lipid backbone on the one hand, and an oligosaccharide moiety on the other hand (Figure 2). While the lipid core is highly conserved across PGL-producing species, the oligosaccharidic domain that is attached to the phenol ring varies $(15,16)$. PGL-bovis, PGL-mar and PGL-ulc from M. bovis, M. marinum and M. ulcerans, respectively, display glycosidic domains that are restricted to a single monosaccharide. Most strains of $M$. tuberculosis, including the laboratory strain H37Rv, do not produce PGL due to a frameshift mutation in the polyketide synthase gene $p k s 15 / 1$ (17). However, strains of the W-Beijing $M$. tuberculosis family have an intact $p k s 15 / 1$, and are known producers of PGL (hereafter named PGL-tb) $(18,19)$. As shown in Figure 2, PGL-tb and PGL-1 of $M$. leprae contain extended, structurally distinct trisaccharidic domains.

\section{PGL and pathogenesis of mycobacterial infections}

The first indication that PGL production may confer additional virulence to mycobacteria came in 2004 with the observation that clinical isolates of $M$. tuberculosis belonging to the W-Beijing family (which express PGL-tb) show a highly lethal phenotype in animal models of infection (18). While hyperpathogenicity was lost upon disruption of PGL production in these isolates, it was not observed with a H37Rv strain engineered to produce PGL-tb (20). This suggested that PGL production is required but not sufficient for M. tuberculosis hyper-pathogenicity. Moreover, it implied that the mycobacterial genetic background interacts with PGL to increase virulence. Notably, ectopic expression of PGL-tb by M. tuberculosis efficiently downregulated the production of inflammatory cytokines in infected monocytes or macrophages, irrespective of the strain used $(18,20)$. Studies in zebrafish showed that PGL expression by $M$. marinum favors the recruitment of macrophages to the site of infection, through 
a host chemokine receptor 2 (CCR2)-mediated pathway (21). Yet, DIM and PGL-mar both contributed to M. marinum virulence in infected zebrafish (22). By applying signature-tag mutagenesis to M. bovis and infection of guinea pigs, Collins et al. identified attenuated mutants with mutated pks 1 , a gene that is essential for PGL biosynthesis (23). PGL-1, made by M. leprae, is detected in all clinical isolates and considered a key player in leprosy pathogenesis $(24,25)$. PGL-1 was indeed shown to mediate the selective tropism of $M$. leprae to Schwann cells, through selective binding of its trisaccharidic moiety to Laminin-2 expressed at the basal lamina (26). More recently, PGL-1 was shown to interact with complement receptor (CR3), either purified and immobilized or displayed at the surface of phagocytes $(9,27)$. Altogether, these studies thus suggest that PGL production contributes to mycobacterial pathogenicity, by directly interfering with host cell invasion and/or by suppressing the generation of innate immune responses by the host. The next section reviews the state of the art with regard to PGL recognition by the innate immune system, and mode of operation.

\section{Molecular mechanisms governing PGL recognition and biological activity}

\subsection{PGL and CR3 signaling}

CR3, also known as Macrophage-1 antigen (MAC-1) is a heterodimeric complex composed of an alpha (CD11b) and beta subunit (CD18). CR3 belongs to the $\beta 2$-integrin family, which also comprises CR4 (CD11c/CD18) and the lymphocyte function-associated antigen 1 (CD11a/CD18) (28). CR3 is a widely expressed receptor found on monocytes, tissue resident macrophages, dendritic cells, neutrophils, NK cells, basophils, eosinophils and platelets. It is also detected on activated CD8 cytotoxic T cells during acute viral infection $(29,30)$ and CD4 T cells $(31)$. Its major functions are cellular adhesion, intracellular signaling and phagocytosis. Notably, CR3 has three conformational states of low-, intermediate- and high-affinity for ligands, co-existing in dynamic equilibrium that can be altered by the activity of other cellular receptors through a process called inside-out signaling. For instance, TLR2 and TLR5 ligation on leukocytes signal to augment $\beta 2$-integrin affinity, resulting in increased cellular adhesion to 
immobilized ICAM-1 and fibronectin (32). CR3 is also able to induce phagocytosis of opsonized and non-opsonized particles, with its I-domain (which binds ICAM-1, fibrinogen and complement component $\mathrm{iC} 3 \mathrm{~b})$ and a carbohydrate-binding lectin domain $(33,34)$.

Phagocytosis of mycobacteria is known to occur in both opsonic and non-opsonic conditions (35), with CR3 being responsible for $40-50 \%$ of non-opsonic binding and $50-60 \%$ opsonic binding of $M$. tuberculosis to macrophages infected in vitro (36). CR3 also mediates the phagocytosis of $M$. leprae by monocyte-derived macrophages in both conditions (37). Also, it was shown that M. bovis BCG promotes its own phagocytic uptake by macrophages, through an inside-out activation of CR3 involving TLR2 (38). While CR3-mediated phagocytosis of mycobacteria occurs irrespective of PGL production, biochemical and cellular evidences suggest that PGL-1 promotes mycobacterial usage of CR3 for phagocyte infection. By designing a recombinant BCG producing PGL-1, Tabouret et al. indeed showed that PGL-1 increases the CR3-dependent uptake of mycobacteria. In line with these findings, PGL-1 was then found to bind CR3 in vitro, through interaction of its sugar moiety with the lectin domain of the receptor (27). Moreover, Lyn, a Src-family tyrosine kinase mediating $\beta 2$-integrin signaling in phagocytes (39), was recently involved in the PGL-1-dependent increase in macrophage invasion (27). Binding to CR3 and exploitation of CR3-mediated phagocytosis was unique to PGL-1, and was not observed with PGL from M. tuberculosis, M. bovis or M. ulcerans. Whether this mechanism involves CD14, TLR2 and associated inside-out activation of CR3 remains unknown.

\subsection{PGL and TLR signaling}

TLR2, TLR4 and TLR9 are activated by mycobacterial products, and functionally important for the control of mycobacterial infection (40). TLR2 KO mice have increased susceptibility to high dose $M$. tuberculosis infection, develop chronic pneumonia and display disrupted granuloma formation (41). In humans, TLR2 receptor polymorphisms are associated with an increased susceptibility in developing 
tuberculosis disease $(42,43)$. TLR4-deficient mice had increased mortality during chronic infection with M. tuberculosis, which was accompanied by reduced IL-12p40, TNF and MCP-1 cytokine production along with reduced macrophage infiltrate in infected lung tissue (44). $M$. leprae stimulates TLR4 in human macrophages that lack TLR2 expression, and blocking TLR4 with neutralizing antibodies before stimulation with killed $M$. leprae resulted in a reduction in CXCL10, IL-6 and TNF- $\alpha$ production (45). Finally, in vivo studies revealed that TLR9 signaling contributes to a regulation of Th1 responses during M. tuberculosis infection (46).

TLR2 recognizes various components of the mycobacterial cell wall, including lipoproteins and glycolipids such as mannose capped-LAM and $\operatorname{LM}(40,47)$. TLR4 recognizes mycobacterial $38 \mathrm{kDa}$ glycoprotein and heat shock protein 60/65, while TLR9 detects unmethylated cytosolic mycobacterial CpG DNA (46). TLR2/4/9 signaling uses the Myeloid Differentiation Primary Response 88 (MyD88) as a common adaptor protein. Other adaptors used by these TLR include TIR domain-containing adaptor protein-inducing IFN- $\beta$ (TRIF), TRIF-related adaptor molecule (TRAM) and TIR-associated protein (TIRAP) (48). The MyD88-dependent pathway signals downstream to activate the transcription factors NF-KB and AP-1, which leads to the transcription of inflammatory cytokines (49). A critical MyD88independent pathway involved in TLR3 and TLR4 signaling utilizes the adaptor protein TRIF to signal downstream to cause the dimerization and activation of IRF3 (50), which results in the transcription of IFN- $\beta$. TRIF also activates the transcription factors $A P-1$ and NF-KB $(51,52)$, a critical requirement for the transcription of inducible nitric oxide synthase (53). Notably, MyD88-deficient mice are able to mount an adaptive immune response to $M$. tuberculosis infection, but succumb more quickly than wild-type mice (54). Although IL-1 receptor signaling is the primary inducer of MyD88-dependent immune responses during the early control of $M$. tuberculosis infection (55), studies in TLR knock-out mice showed that TLR-mediated signals are required for the long-term control of infection, through 
initiating bactericidal functions and triggering the production of pro-inflammatory cytokines $(41,44$, 46).

Notably, PGL-1 and PGL-tb analogues missing part or all of the lipid core inhibited the activation of NFKB and downstream production of inflammatory cytokines by TLR2-stimulated macrophages (27, $56,57)$. In line with this result, native PGL-1 was recently found to bind TLR2 in vitro, through its trisaccharidic epitope (27). Differently from CR3-mediated phagocytosis, PGL-1-mediated inhibition of TLR2 stimulation was not affected by Lyn silencing. Moreover, while PGL-1 was unique among PGL in its capacity to promote CR3-mediated phagocytosis, TLR2 signaling was comparably inhibited by PGL1 and PGL-tb. In contrast, monosaccharide-bearing PGL did not display such an effect. Together, these observations thus suggest that PGL-1 and PGL-tb inhibit TLR2 activation independently of CR3 signaling.

Parallel to this, it was shown that in vitro exposure of monocytes from healthy donors to PGL-1 interferes with their production of TNF- $\alpha$ following stimulation with the TLR4 agonist LPS and IFN- $\gamma$ (58). Tabouret et al. reported previously that bacterially-expressed PGL-1 alters the induction of NF$\kappa B$ and subsequent TNF- $\alpha$ production by infected macrophages, in a CR3-dependent manner (9). More recently, we found that PGL-1 (and PGL-tb) also impairs the induction of nitric oxide synthase (iNOS), and subsequent production of bactericidal nitric oxide (NO) by macrophages stimulated with LPS and IFN- $\gamma$, in a CR3-dependent manner (unpublished observations). We found that PGL-1 and PGL-tb operate by downregulating the endogenous levels of TRIF in macrophages. Notably, PGL-mediated decrease in TRIF protein levels occurred post-transcriptionally and independently of Src-family tyrosine kinases, lysosomal and proteasomal degradation. We observed a selective impairment of the induction of TRIF-dependent gene products, including NO synthase and inflammatory mediators IFN$\beta$ and CXCL10, upon cell stimulation of TLR4. With TRIF being situated at the crossroads of NO and cytokine production, this observation thus provided a unifying mechanism for the capacity of PGL to 
promote mycobacterial intracellular persistence while dampening inflammatory signaling. To conclude, PGL-1 and PGL-tb are capable of suppressing TLR2 and TLR4 signaling in CR3-independent and CR3dependent manners, respectively. Our observation that PGL-1 and PGL-tb induce TRIF downregulation suggest that they may also affect TLR3 signaling, and therefore the host response during viral coinfection.

\section{Conclusion}

Thanks to recent advances in the genetic engineering of mycobacteria and in synthetic glycobiology, our understanding of the molecular mechanisms underlying the pathogenic and immunosuppressive properties of PGL has significantly progressed over the past few years. Studies using synthetic subunits of PGL and recombinant BCG expressing species-specific PGL have allowed to show that certain PGL interact with multiple pattern recognition receptors expressed by host phagocytes and alter their intracellular dialogue (Figure 3). The unique oligosaccharide domain of PGL-1, made by M. leprae, was shown to hijack CR3 for optimal invasion of host phagocytes. In addition, PGL-1 and PGL-tb (produced by highly pathogenic clinical isolates of $M$. tuberculosis), but not PGL-bovis or PGL-ulc, shared the capacity to block agonist-driven activation of TLR2. Additionally, these PGL downregulated TRIFdependent TLR4 signaling in macrophages, producing relatively less inflammatory and bactericidal responses during infection. Since $M$. tuberculosis and $M$. leprae are the most successful pathogens in the Mycobacterium genus, it is tempting to speculate that they have evolved the biosynthesis of PGL with extended saccharidic domains to escape from the host immune system. Much remains to be elucidated, such as the fine structural specificities of PGL binding to CR3 and TLR2, the contribution of inside-out signaling in PGL binding to CR3, and the CR3-dependent mechanism that leads to TRIF downregulation. The answers to these questions will not only improve our understanding of mycobacterial pathogenicity, but will also undoubtedly reveal novel mechanisms that regulate inflammation by modifying the crosstalk between innate immunity receptors. 


\section{Acknowledgments}

This study was supported by the "Marie Skłodowska-Curie Actions" of the European Union's Seventh Framework Programme FP7/2007-2013, REA grant agreement $n^{\circ} 317057$. We thank Jacques Prandi (CNRS, IPBS, Toulouse, France) for drawing the chemical structures shown in Figure 2.

\section{References}

1. Rastogi N, Legrand E, Sola C. The mycobacteria: an introduction to nomenclature and pathogenesis. Rev Sci Tech. 2001;20(1):21-54.

2. Springer B, Stockman L, Teschner K, Roberts GD, Bottger EC. Two-laboratory collaborative study on identification of mycobacteria: Molecular versus phenotypic methods. Journal of Clinical Microbiology. 1996;34(2):296-303.

3. Springer B, Wu WK, Bodmer T, Haase G, Pfyffer GE, Kroppenstedt RM, et al. Isolation and characterization of a unique group of slowly growing mycobacteria: Description of Mycobacterium lentiflavum sp nov. Journal of Clinical Microbiology. 1996;34(5):1100-7.

4. Brosch R, Gordon SV, Marmiesse M, Brodin P, Buchrieser C, Eiglmeier K, et al. A new evolutionary scenario for the Mycobacterium tuberculosis complex. Proc Natl Acad Sci U S A. 2002;99(6):36849.

5. Esmail H, Barry CE, 3rd, Young DB, Wilkinson RJ. The ongoing challenge of latent tuberculosis. Philos Trans R Soc Lond B Biol Sci. 2014;369(1645):20130437.

6. Behr MA, Small PM. A historical and molecular phylogeny of BCG strains. Vaccine. 1999;17(78):915-22.

7. Brosch R, Pym AS, Gordon SV, Cole ST. The evolution of mycobacterial pathogenicity: clues from comparative genomics. Trends Microbiol. 2001;9(9):452-8. 
8. Mahairas GG, Sabo PJ, Hickey MJ, Singh DC, Stover CK. Molecular analysis of genetic differences between Mycobacterium bovis BCG and virulent M. bovis. J Bacteriol. 1996;178(5):1274-82.

9. Tabouret G, Astarie-Dequeker C, Demangel C, Malaga W, Constant P, Ray A, et al. Mycobacterium leprae phenolglycolipid-1 expressed by engineered M. bovis BCG modulates early interaction with human phagocytes. PLoS Pathog. 2010;6(10):e1001159.

10. Hoffmann C, Leis A, Niederweis M, Plitzko JM, Engelhardt H. Disclosure of the mycobacterial outer membrane: cryo-electron tomography and vitreous sections reveal the lipid bilayer structure. Proc Natl Acad Sci U S A. 2008;105(10):3963-7.

11. Sani M, Houben EN, Geurtsen J, Pierson J, de Punder K, van Zon M, et al. Direct visualization by cryo-EM of the mycobacterial capsular layer: a labile structure containing ESX-1-secreted proteins. PLoS Pathog. 2010;6(3):e1000794.

12. Zuber B, Chami M, Houssin C, Dubochet J, Griffiths G, Daffe M. Direct visualization of the outer membrane of mycobacteria and corynebacteria in their native state. J Bacteriol. 2008;190(16):5672-80.

13. Watanabe M, Yamada Y, Iguchi K, Minnikin DE. Structural elucidation of new phenolic glycolipids from Mycobacterium tuberculosis. Biochim Biophys Acta. 1994;1210(2):174-80.

14. Onwueme KC, Vos CJ, Zurita J, Ferreras JA, Quadri LE. The dimycocerosate ester polyketide virulence factors of mycobacteria. Prog Lipid Res. 2005;44(5):259-302.

15. Arbues A, Lugo-Villarino G, Neyrolles O, Guilhot C, Astarie-Dequeker C. Playing hide-and-seek with host macrophages through the use of mycobacterial cell envelope phthiocerol dimycocerosates and phenolic glycolipids. Front Cell Infect Microbiol. 2014;4:173.

16. Guenin-Mace L, Simeone R, Demangel C. Lipids of pathogenic Mycobacteria: contributions to virulence and host immune suppression. Transbound Emerg Dis. 2009;56(6-7):255-68.

17. Constant P, Perez E, Malaga W, Laneelle MA, Saurel O, Daffe M, et al. Role of the pks $15 / 1$ gene in the biosynthesis of phenolglycolipids in the Mycobacterium tuberculosis complex. Evidence that all strains synthesize glycosylated p-hydroxybenzoic methyl esters and that strains devoid of 
phenolglycolipids harbor a frameshift mutation in the pks15/1 gene. J Biol Chem. $2002 ; 277(41): 38148-58$

18. Reed MB, Domenech P, Manca C, Su H, Barczak AK, Kreiswirth BN, et al. A glycolipid of hypervirulent tuberculosis strains that inhibits the innate immune response. Nature. 2004;431(7004):84-7.

19. Tsenova L, Ellison E, Harbacheuski R, Moreira AL, Kurepina N, Reed MB, et al. Virulence of selected Mycobacterium tuberculosis clinical isolates in the rabbit model of meningitis is dependent on phenolic glycolipid produced by the bacilli. J Infect Dis. 2005;192(1):98-106.

20. Sinsimer D, Huet G, Manca C, Tsenova L, Koo MS, Kurepina N, et al. The phenolic glycolipid of Mycobacterium tuberculosis differentially modulates the early host cytokine response but does not in itself confer hypervirulence. Infect Immun. 2008;76(7):3027-36.

21. Cambier CJ, Takaki KK, Larson RP, Hernandez RE, Tobin DM, Urdahl KB, et al. Mycobacteria manipulate macrophage recruitment through coordinated use of membrane lipids. Nature. $2014 ; 505(7482): 218-22$

22. Yu J, Tran V, Li M, Huang X, Niu C, Wang D, et al. Both phthiocerol dimycocerosates and phenolic glycolipids are required for virulence of Mycobacterium marinum. Infect Immun. 2012;80(4):1381-9.

23. Collins DM, Skou B, White S, Bassett S, Collins L, For R, et al. Generation of attenuated Mycobacterium bovis strains by signature-tagged mutagenesis for discovery of novel vaccine candidates. Infect Immun. 2005;73(4):2379-86.

24. Hunter SW, Brennan PJ. A novel phenolic glycolipid from Mycobacterium leprae possibly involved in immunogenicity and pathogenicity. J Bacteriol. 1981;147(3):728-35.

25. Cho SN, Hunter SW, Gelber RH, Rea TH, Brennan PJ. Quantitation of the phenolic glycolipid of Mycobacterium leprae and relevance to glycolipid antigenemia in leprosy. J Infect Dis. 1986;153(3):560-9. 
26. Ng V, Zanazzi G, Timpl R, Talts JF, Salzer JL, Brennan PJ, et al. Role of the cell wall phenolic glycolipid-1 in the peripheral nerve predilection of Mycobacterium leprae. Cell. 2000;103(3):51124.

27. Arbues A, Malaga W, Constant P, Guilhot C, Prandi J, Astarie-Dequeker C. Trisaccharides of Phenolic Glycolipids Confer Advantages to Pathogenic Mycobacteria through Manipulation of Host-Cell Pattern-Recognition Receptors. ACS Chem Biol. 2016.

28. Rosetti F, Mayadas TN. The many faces of Mac-1 in autoimmune disease. Immunol Rev. $2016 ; 269(1): 175-93$.

29. Christensen JE, Andreasen SO, Christensen JP, Thomsen AR. CD11b expression as a marker to distinguish between recently activated effector CD8(+) T cells and memory cells. Int Immunol. 2001;13(4):593-600.

30. Fiorentini S, Licenziati S, Alessandri G, Castelli F, Caligaris S, Bonafede M, et al. CD11b expression identifies CD8+CD28+ T lymphocytes with phenotype and function of both naive/memory and effector cells. J Immunol. 2001;166(2):900-7.

31. Erdei A, Sandor N, Macsik-Valent B, Lukacsi S, Kremlitzka M, Bajtay Z. The versatile functions of complement C3-derived ligands. Immunol Rev. 2016;274(1):127-40.

32. Chung KJ, Mitroulis I, Wiessner JR, Zheng YY, Siegert G, Sperandio M, et al. A novel pathway of rapid TLR-triggered activation of integrin-dependent leukocyte adhesion that requires Rap1 GTPase. Mol Biol Cell. 2014;25(19):2948-55.

33. Diamond MS, Garcia-Aguilar J, Bickford JK, Corbi AL, Springer TA. The I domain is a major recognition site on the leukocyte integrin Mac-1 (CD11b/CD18) for four distinct adhesion ligands. J Cell Biol. 1993;120(4):1031-43.

34. O'Brien XM, Heflin KE, Lavigne LM, Yu K, Kim M, Salomon AR, et al. Lectin site ligation of CR3 induces conformational changes and signaling. J Biol Chem. 2012;287(5):3337-48. 
35. Le Cabec V, Carreno S, Moisand A, Bordier C, Maridonneau-Parini I. Complement receptor 3 (CD11b/CD18) mediates type I and type II phagocytosis during nonopsonic and opsonic phagocytosis, respectively. J Immunol. 2002;169(4):2003-9.

36. Melo MD, Catchpole IR, Haggar G, Stokes RW. Utilization of CD11b knockout mice to characterize the role of complement receptor $3(\mathrm{CR} 3, \mathrm{CD} 11 \mathrm{~b} / \mathrm{CD} 18)$ in the growth of Mycobacterium tuberculosis in macrophages. Cell Immunol. 2000;205(1):13-23.

37. Schlesinger LS, Horwitz MA. Phagocytosis of Mycobacterium leprae by human monocyte-derived macrophages is mediated by complement receptors CR1 (CD35), CR3 (CD11b/CD18), and CR4 (CD11C/CD18) and IFN-gamma activation inhibits complement receptor function and phagocytosis of this bacterium. J Immunol. 1991;147(6):1983-94.

38. Sendide K, Reiner NE, Lee JS, Bourgoin S, Talal A, Hmama Z. Cross-talk between CD14 and complement receptor 3 promotes phagocytosis of mycobacteria: regulation by phosphatidylinositol 3-kinase and cytohesin-1. J Immunol. 2005;174(7):4210-9.

39. Adachi R, Suzuki K. Lyn, one of the Src-family tyrosine kinases expressed in phagocytes, plays an important role in beta2 integrin-signalling pathways in opsonized zymosan-activated macrophage-like U937 cells. Cell Biochem Funct. 2007;25(3):323-33.

40. Saraav I, Singh S, Sharma S. Outcome of Mycobacterium tuberculosis and Toll-like receptor interaction: immune response or immune evasion? Immunol Cell Biol. 2014;92(9):741-6.

41. Drennan MB, Nicolle D, Quesniaux VJ, Jacobs M, Allie N, Mpagi J, et al. Toll-like receptor 2deficient mice succumb to Mycobacterium tuberculosis infection. Am J Pathol. 2004;164(1):4957.

42. Ogus AC, Yoldas B, Ozdemir T, Uguz A, Olcen S, Keser I, et al. The Arg753GLn polymorphism of the human toll-like receptor 2 gene in tuberculosis disease. Eur Respir J. 2004;23(2):219-23.

43. Ben-Ali M, Barbouche MR, Bousnina S, Chabbou A, Dellagi K. Toll-like receptor 2 Arg677Trp polymorphism is associated with susceptibility to tuberculosis in Tunisian patients. Clin Diagn Lab Immunol. 2004;11(3):625-6. 
44. Abel B, Thieblemont N, Quesniaux VJ, Brown N, Mpagi J, Miyake K, et al. Toll-like receptor 4 expression is required to control chronic Mycobacterium tuberculosis infection in mice. J Immunol. 2002;169(6):3155-62.

45. Polycarpou A, Holland MJ, Karageorgiou I, Eddaoudi A, Walker SL, Willcocks S, et al. Mycobacterium leprae Activates Toll-Like Receptor-4 Signaling and Expression on Macrophages Depending on Previous Bacillus Calmette-Guerin Vaccination. Front Cell Infect Microbiol. $2016 ; 6: 72$.

46. Bafica A, Scanga CA, Feng CG, Leifer C, Cheever A, Sher A. TLR9 regulates Th1 responses and cooperates with TLR2 in mediating optimal resistance to Mycobacterium tuberculosis. J Exp Med. $2005 ; 202(12): 1715-24$.

47. Krutzik SR, Ochoa MT, Sieling PA, Uematsu S, Ng YW, Legaspi A, et al. Activation and regulation of Toll-like receptors 2 and 1 in human leprosy. Nat Med. 2003;9(5):525-32.

48. Kawai T, Akira S. Toll-like receptors and their crosstalk with other innate receptors in infection and immunity. Immunity. 2011;34(5):637-50.

49. Akira S, Takeda K. Toll-like receptor signalling. Nat Rev Immunol. 2004;4(7):499-511.

50. Noyce RS, Collins SE, Mossman KL. Differential modification of interferon regulatory factor 3 following virus particle entry. J Virol. 2009;83(9):4013-22.

51. O'Neill LA, Golenbock D, Bowie AG. The history of Toll-like receptors - redefining innate immunity. Nat Rev Immunol. 2013;13(6):453-60.

52. Hoebe K, Du X, Georgel P, Janssen E, Tabeta K, Kim SO, et al. Identification of Lps2 as a key transducer of MyD88-independent TIR signalling. Nature. 2003;424(6950):743-8.

53. Hoebe K, Janssen EM, Kim SO, Alexopoulou L, Flavell RA, Han J, et al. Upregulation of costimulatory molecules induced by lipopolysaccharide and double-stranded RNA occurs by Trifdependent and Trif-independent pathways. Nat Immunol. 2003;4(12):1223-9. 
54. Fremond CM, Yeremeev V, Nicolle DM, Jacobs M, Quesniaux VF, Ryffel B. Fatal Mycobacterium tuberculosis infection despite adaptive immune response in the absence of MyD88. J Clin Invest. $2004 ; 114(12): 1790-9$

55. Fremond CM, Togbe D, Doz E, Rose S, Vasseur V, Maillet I, et al. IL-1 receptor-mediated signal is an essential component of MyD88-dependent innate response to Mycobacterium tuberculosis infection. J Immunol. 2007;179(2):1178-89.

56. Elsaidi HR, Barreda DR, Cairo CW, Lowary TL. Mycobacterial phenolic glycolipids with a simplified lipid aglycone modulate cytokine levels through Toll-like receptor 2 . Chembiochem. 2013;14(16):2153-9.

57. Elsaidi HR, Lowary TL. Inhibition of cytokine release by mycobacterium tuberculosis phenolic glycolipid analogues. Chembiochem. 2014;15(8):1176-82.

58. Fallows D, Peixoto B, Kaplan G, Manca C. Mycobacterium leprae alters classical activation of human monocytes in vitro. J Inflamm (Lond). 2016;13:8. 


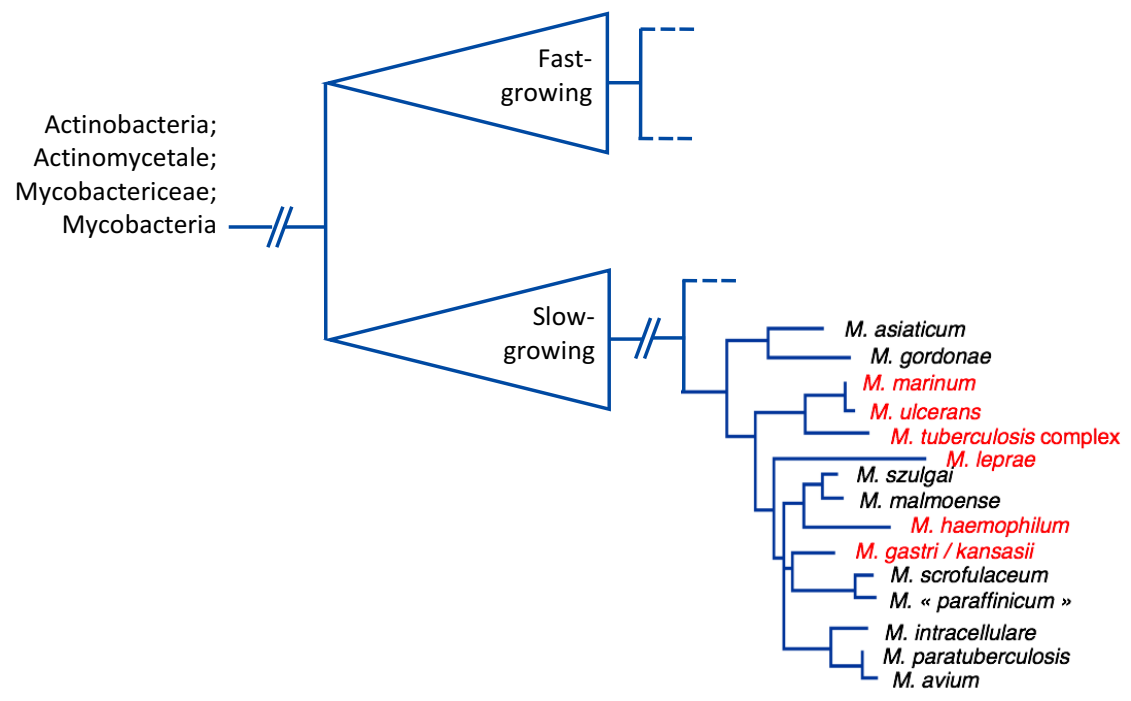

Figure 1: Phylogenetic position of the PGL-producing species within the genus Mycobacterium. PGL-producing species are shown in red, in the context of their phylogenetic branch, in the subfamily of slow-growing mycobacteria. Adapted from Springer et al., 1996 (2, 3). 

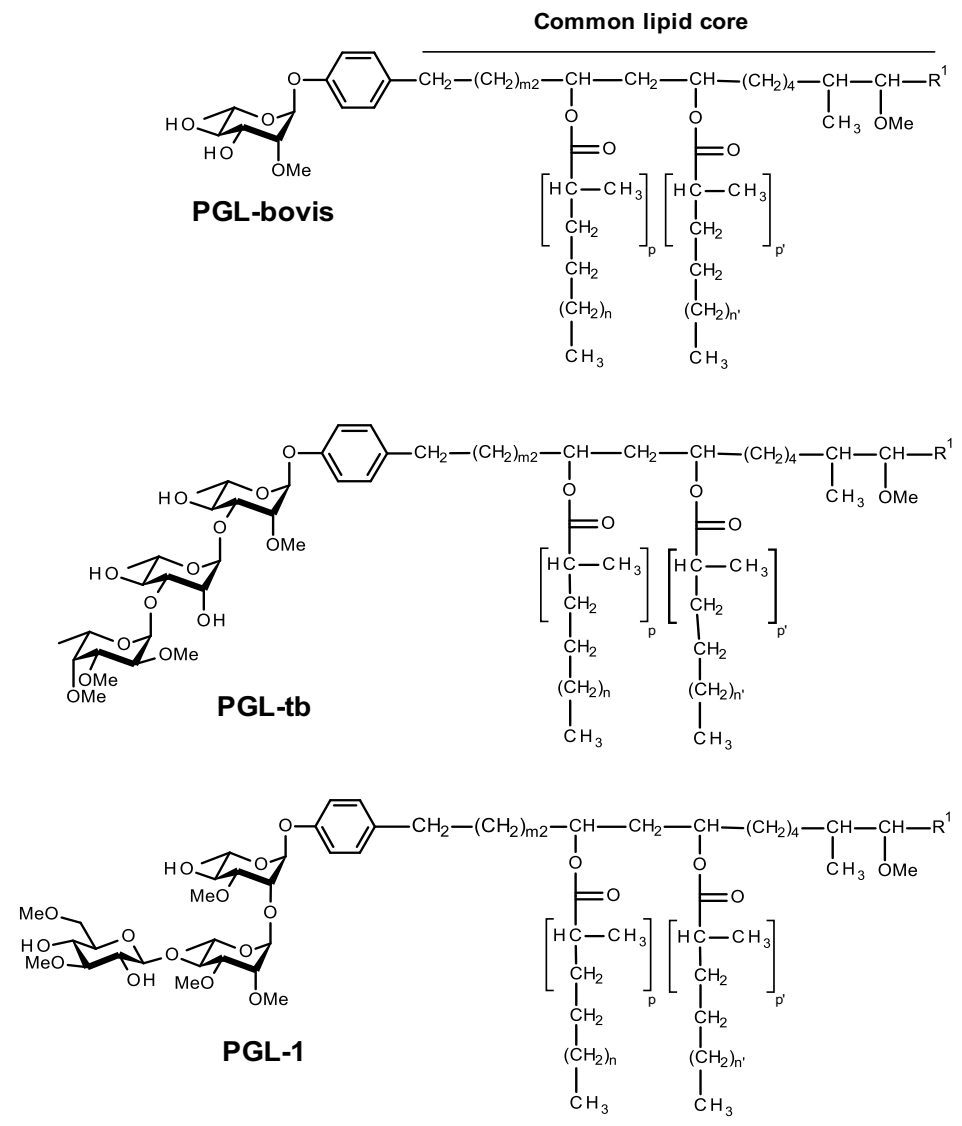

$\mathrm{m} 1=16-22, \mathrm{~m} 2=13-17$

$\mathrm{n}, \mathrm{n}^{\prime}=15-17, \mathrm{p}, \mathrm{p}^{\prime}=3-5$

$\mathrm{R}^{1}=\mathrm{CH}_{3}$ or $\mathrm{CH}_{2} \mathrm{CH}_{3}$

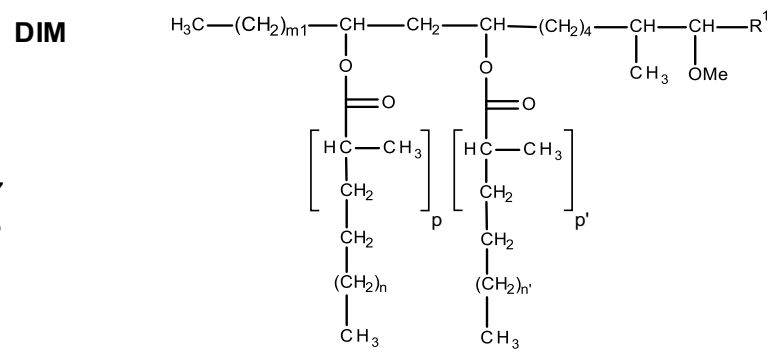

Figure 2: Structure of the major forms of PGL made by Mycobacterium bovis (PGL-bovis), $M$. tuberculosis (PGL-tb) and M. leprae (PGL-1), and of mycobacterial DIM. The common lipid core is composed of a long-chain $\beta$-diol esterified by polymethyl-branched fatty acids. Adapted from Arbues et al., 2014 (15). 


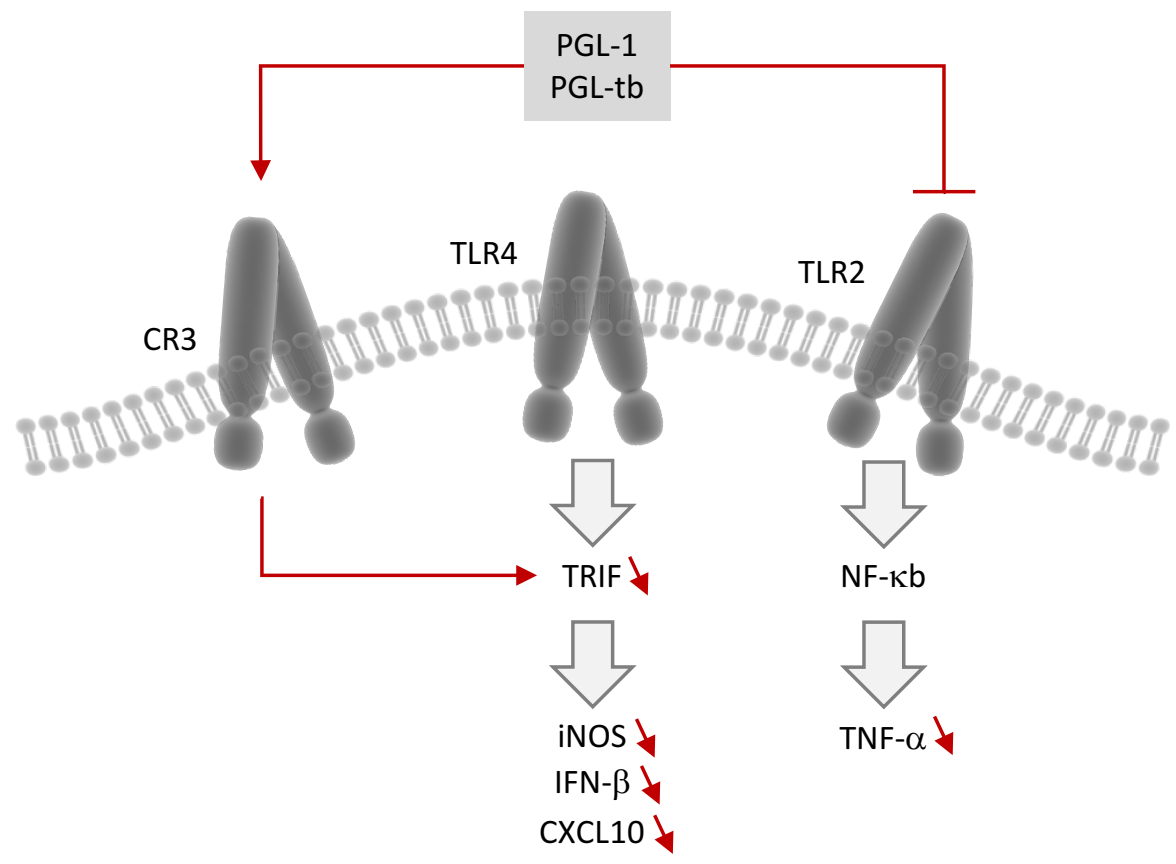

Figure 3: Schematic diagram recapitulating the mechanisms used by PGL-1 and PGL-tb to suppress the innate immune responses of macrophages 